\title{
Numerical Flow Analysis of a Centrifugal Compressor with Ported and without Ported Shroud
}

\author{
Bernhard Semlitsch, JyothishKumar V , Mihai Mihaescu and Laszlo Fuchs \\ KTH Royal Institute of Technology \\ Matthieu Gancedo and Ephraim Gutmark \\ UC University of Cincinnati
}

Copyright $@ 2014$ SAE International

\begin{abstract}
Turbochargers are commonly used in automotive engines to increase the internal combustion engine performance during off design operation conditions. When used, a most wide operation range for the turbocharger is desired, which is limited on the compressor side by the choke condition and the surge phenomenon. The ported shroud technology is used to extend the operable working range of the compressor, which permits flow disturbances that block the blade passage to escape and stream back through the shroud cavity to the compressor inlet. The impact of this technology on a speed-line at near optimal operation condition and near surge operation condition is investigated.
\end{abstract}

A numerical study investigating the flow-field in a centrifugal compressor of an automotive turbocharger has been performed using Large Eddy Simulation. The wheel rotation is handled by the numerically expensive sliding mesh technique. In this analysis, the full compressor geometry (360 deg) is considered. Numerical solutions with and without ported shroud for a near optimal operation condition and near-surge operation condition. The flow-field of the different cases is analyzed to elucidate the functionality of the ported shroud. In agreement with previous observations, it was found that the ported shroud reduces the flow disturbances in the blade passage for all operating conditions. However, the compressor efficiency for the off-design operation condition was found to be higher without the ported shroud, supporting the findings reported recently by an experimental investigation. The computational results are validated with experimental measurements in terms of the performance parameters and available Particle Image Velocimetry data.

\section{Introduction}

Due to the regular reinforcement of emission regulations, engine downsizing has become a common practice to increase the specific power and the efficiency of Internal Combustion (IC) engines. The use of turbochargers allows to increase the amount of charge delivered into the combustion cylinder, which leads to more amount of fuel that can be burnt and thus, more power can be generated. Hence, by using this technology in a combination with a smaller cylinder an equal amount of power can be generated, when the work performed by the turbocharger is exploited, as with an engine consisting of a larger cylinder without turbocharger. Commonly, the turbocharger comprises of a radial turbine and a centrifugal compressor mounted on the same shaft. The compressor increases the intake density of the charge and therefore, increases the amount of loading. The compressor is driven by the turbine, which exploits the energy of the hot exhaust gases after combustion. Therefore, the total efficiency of the engine is increased at the off-design conditions, by maximizing the amount of extracted energy from the fuel. Furthermore, the efficient operating range of the IC engine is increased using a turbocharger, which leads to a reduction of emissions in particular for Diesel engines.

The centrifugal compressor in turbochargers is required to condense the charge before the engine cylinder. However, during a driving cycle the automotive compressor has to operate efficiently over a wide operating range of mass-flow rates. Diesel engines with high pressure-ratio turbochargers operate the centrifugal compressor close to the occurrence of the surge instability. With the surge phenomenon, high pressure fluctuations occur in the blade passage, which induce high stresses in the blades and this can lead to fatigue of the compressor. The occurrence of the surge phenomenon is a function of the rotational speed of the centrifugal compressor and it is difficult to predict the onset of this phenomenon accurately. Since surge is not completely understood, it requires an effort to improve the working range of the centrifugal compressor without compromising the pressureratio or the compressor efficiency.

There are mainly two methods to accomplish the task of increasing the width of the compressor map, i.e. using active flow control or using passive flow control devices. Active flow control devices include e.g. the variable vaned diffusers, and the variable inlet guide vanes [1-4]. Passive flow control devices including casing treatment [5-8], (which includes the ported shroud,) inducer casing bleed system [7-9], and internal recirculation or ring groove arrangements [10-12]. From the above mentioned devices, the variable inlet guide vanes and variable vaned diffuser introduce an efficiency penalty along with a gain of wider compressor operability range. However, 
turbochargers are required to be compact, producible and operational at low cost. Therefore, only simple designs with good reliability are adopted and realized. The implementation of these active flow control devices is very complicated on existing compressor designs. However, the passive flow control devices like ported shroud casings are very simple, easy to implement and therefore they are largely used in the centrifugal compressor of turbochargers. With the ported shroud, a part of the low momentum flow blocking the wheel is transferred back to the compressor intake and the blade passage is cleaned from flow disturbances. Thereby the working range of the compressor is extended. The ported shroud operation sketch is shown in Fig. 1. Thus, the passive flow control device becomes a means of improving the working width of the compressor.

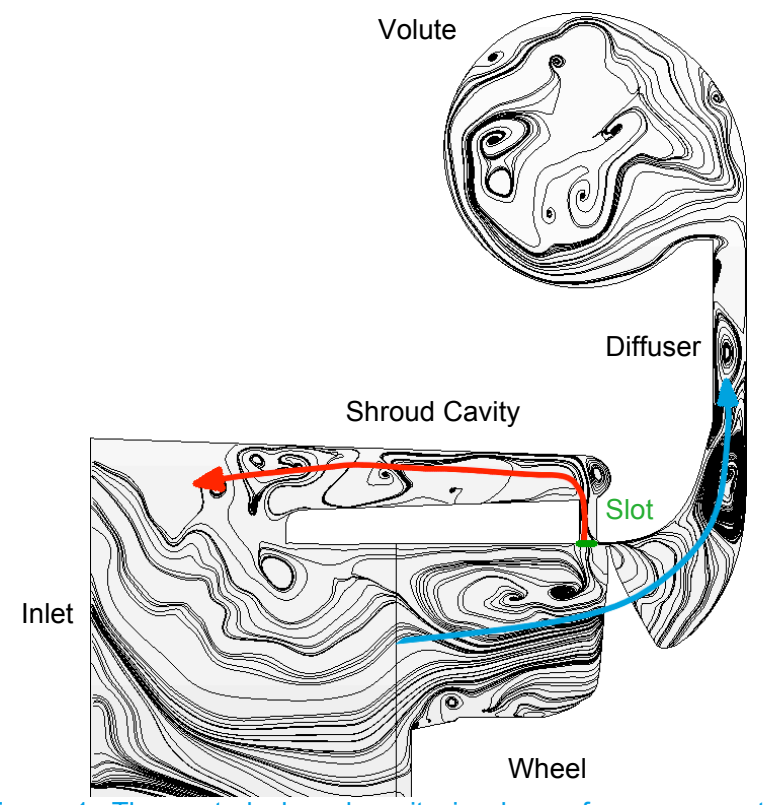

Figure 1. The ported shroud cavity is shown for a segment of the compressor, where the slot entrance is colored in green. The sketch illustrates the flow path for normal operation (blue) and flow recirculation (red).

Numerous of investigations have been carried out to improve the operating range of centrifugal compressors. Fisher [13] performed experimental research on self-recirculation casing treatment on a centrifugal turbocharger compressor. It has been observed that the pressure gradient between the slots drives the flow through the shroud cavity. The near-stall condition of the compressor caused a low momentum flow near the shroud side, which was forced to stream through the shroud cavity towards the main inlet due to the favorable pressure gradient. Numerical and experimental studies have been carried out by Hunziker et. al. [14] to understand the effect of inducer casing bleed system. The numerical calculations were carried out for different configurations and a promising configuration showed a significant extension increase of the compressor operation map width without affecting the efficiency. Several studies were carried out with vanes installed inside of the ported shroud $[7,10,11]$. It was found that the inclined vane inserts reduce the surge flow rate by $20 \%$ when compared to traditional ported shroud configurations.
Several Large Eddy Simulation (LES) investigations have been performed analyzing the flow phenomena occurring in centrifugal compressors $[11,12,15]$. Flow separation and recirculation bubbles were accurately predicted and the LES approach results were more accurate in predicting the performance parameters and the flow structure details than Reynolds Averaged Navier-Stokes (RANS) simulations. However, these studies were limited to the application of rotating reference frame to model the rotational effect of the impeller. With LES the unsteady flow phenomena can be studied and the induced flow frequencies are simulated accurately. The studies were carried out near the optimal efficiency operating condition and near-surge operating conditions to analyze the turbulent flow-field development inside the compressor domain. Using a time-averaged approach as e.g. RANS, the unsteady large scale structures and their effect on turbulence cannot be handled, as RANS models are calibrated for statistically stationary flows. LES, on the other hand, handles all the larger scales as well as parts of the turbulent spectrum that are affected by the large nonturbulent structures and their interaction with the larger turbulent scales. The smaller scales of turbulence, given adequate resolution, are of universal character and hence independent of the flow geometry and the imposed boundary conditions, not too close to the boundaries. Thus with LES, on may quantify the propagation of disturbances at the boundaries into the impeller and thereby also enable the understanding of the processes that may lead to surge and stall. Previous studies have been focusing on single blade passages and conclusions have been drawn on the flow through the impeller.

In the present work, LES computations of the flow inside a turbocharger centrifugal compressor with ported and nonported shroud configurations at near optimal efficiency operation condition and near-surge operation conditions are performed, where the LES approach has been used. The full 360-degree geometry is simulated, where the rotation of the impellor mesh is handled by the sliding mesh technique. The numerically obtained performance parameters are validated using experimental measurements and the flow field at the compressor inlet is compared to experimental flow visualizations.

\section{Geometry and Flow Modeling}

The geometry used for the numerical simulation analysis is a ported shroud compressor of an automotive turbocharger, as illustrated in Fig. 2a. The inlet and outlet boundaries are indicated. The ported shroud cavity is shown in Fig. 2b separately to indicate the asymmetric arrangement of the four rips separating the ported shroud cavities. Through this ported shroud cavity, some flow is allowed to recirculate back to the compressor inlet. The non-ported shroud configuration is obtained by closing numerically the slot entrance of the ported shroud on the shroud side of the wheel passage, which is shown in green color in Fig. 1 and in yellow color in Fig. 2. The compressor specifications are shown in Tab. 1. 




(a) Full Compressor Domain



(c) Full Compressor Domain

Figure 2. Used geometry of the centrifugal compressor.

Table 1. Compressor design details.

\begin{tabular}{|l|l|}
\hline Turbocharger compatibility & Heavy truck engine \\
\hline Engine power range & 400 to $850 \mathrm{~kW}$ \\
\hline Number of blades & 10 \\
\hline Exducer diameter & $88 \mathrm{~mm}$ \\
\hline TRIM & 56 \\
\hline Diffuser area ratio & 0.57 \\
\hline
\end{tabular}

A polygonal mesh grid was used for numerical discretization of the computational domain. Nevertheless, a structured of blockstructured grid exhibits advantages in numerical accuracy and one could better control of the mesh cell distribution. On the other hand, a good structured mesh grid requires much human effort for complex geometries as compared to an unstructured grid. In a complex geometry it is sometimes unavoidable to skew heavily the mesh cell or to use large aspect ratio in certain regions, which may lead to a local decrease of accuracy. Therefore, an unstructured, auto-generated mesh with a total number of cells of about 6 million was preferred. Using an auto-generated mesh, this type of mesh grid is easy to generate and has less diffusive errors, i.e. better accuracy, compared to an equivalent tetrahedral mesh. The polygonal mesh results in improving the numerical accuracy for complex geometry when compared to other types of unstructured meshes [16]. The wheel rotation is handled by the sliding mesh technique. With this technique, the impeller is represented by a set of grids rotating relative to the stationary grid of the domain. At the interface between the rotating and stationary part, the moving mesh slides over the stationary mesh and the primary variables are propagated over this interface. At each time step, the cell connectivity of the numerical grid changes on either side of the interface.

The equations governing the compressible flow are the conservation equations of continuity, momentum, and energy. This set of equations is solved numerically along with the equation of state, i.e. the ideal gas law, using a commercial code STARCCM+ [17] based on the finite volume method. The time integration of the equations is carried out using an implicit second-order accurate scheme, where a time step of $1.3 \cdot 10^{-5} \mathrm{~s}$ was chosen for time advancement of the transient simulation. This rather high value was selected such that the inherent high frequencies of this problem (blade passing frequency $10.6 \mathrm{kHz}$ ) can be resolved and the low frequent flow phenomena in the range $10 \mathrm{~Hz}-50 \mathrm{~Hz}$ (related to surge phenomenon) can be resolved with a reasonable numerical effort, since the focus of this study is the low frequency range. A hybrid second order bounded central differencing scheme is used for spatial discretization. The scheme uses a blend of second and first order upwind scheme for numerical stabilization when the boundedness of the central differencing scheme is not guarantied.

The LES approach is used for handling turbulence. The large turbulent flow scales are dependent on the boundary conditions and the geometrical shape of the domain. Thus, the large turbulent flow scales are governing the overall behavior of the flow and it is relevant to explicitly capture these flowscales within the numerical grids. The small-scale turbulent flow structures are dissipating the turbulent kinetic energy transferred from the large energetic turbulent flow scales. The dissipation of flow energy to heat at small flow scales is independent of the geometry. Using the LES approach, the numerical grid does not resolve these small flow scales and therefore these scales are called the Sub-Grid Scales (SGS). Applying some assumptions of the energy dissipation behavior, the dissipative behavior can be modeled. In the present LES computations there is no explicit SGS model used. An implicit LES approach has been used for the flow computation. This approach takes into account the inherent numerical dissipation of the differencing scheme used for the finite volume discretization.

The simulations are carried out at near optimal efficiency operation condition and at near surge conditions for which experimental data have been obtained at the Gas Dynamics and Propulsion Laboratory of the University of Cincinnati. In all computed cases, the mass flow rate at inlet plane of the compressor is specified as the inflow boundary condition, along with the static pressure imposed at the exit plane. The maximum efficiency condition is different for the cases with and without ported shroud. Thus, a representative case, near optimal conditions has been chosen. For the near optimal operating efficiency condition, a mass flow rate of $0.27 \mathrm{~kg} / \mathrm{s}$ has been set and an exit static pressure of $156 \mathrm{kPa}$ has been applied. For the near surge operation condition, the mass flow rate has been lowered to a value of $0.105 \mathrm{~kg} / \mathrm{s}$, while the exit static pressure has been increased to $165 \mathrm{kPa}$. The inlet total temperature of $296 \mathrm{~K}$ is specified for all conditions. The rotational speed of the compressor is set to $64000 \mathrm{rpm}$ for all treated cases. No-slip boundary conditions are specified at all walls and the walls are considered to be smooth and adiabatic. 


\section{Results}

The result section of the paper is divided into three sections: (i) validation - for the ported and non-ported shroud compressor and the comparison with experimental data, (ii) general flow field comparison between the two configurations, and (iii) frequency spectra analysis of the flow structures.

\section{(i) Validation}

A comparison has been made between the ported and nonported shroud compressor with respect to the pressure-ratio and the compressor efficiency at near optimal operating conditions and near-surge. The numerically obtained data is tabulated in Tab. 2, together with the experimental data measured at University of Cincinnati [18] for the ported and non-ported shroud compressor. The simulation results are in agreement with experimental data. The numerical simulations capture the compressor efficiency trends consistently. However, the computations predict different pressure ratios for the non-ported shroud case near surge. The pressure-ratios obtained from computations are illustrated on the experimental compressor map shown in Fig. 3, where the experimental data was plotted as function of the corrected mass-flow rate. A vertical dashed line in Fig. 3 indicates the surge line.

Table 2. Numerical and experimental data comparison.

\begin{tabular}{|c|c|c|c|c|c|}
\hline \multirow{2}{*}{ Cases } & \multirow{2}{*}{ Operating Condition } & \multicolumn{2}{|c|}{ Pr. } & \multicolumn{2}{c|}{ Efficiency $(\eta)$} \\
\cline { 3 - 6 } & & LES & Exp. & LES & Exp. \\
\hline \multirow{2}{*}{ Ported Shroud } & Near Optimal Efficiency & 1.59 & 1.52 & 0.74 & 0.70 \\
\cline { 2 - 6 } & Near-Surge & 1.68 & 1.66 & 0.55 & 0.58 \\
\hline \multirow{2}{*}{$\begin{array}{c}\text { Non-ported } \\
\text { Shroud }\end{array}$} & Near Optimal Efficiency & 1.59 & 1.52 & 0.74 & 0.72 \\
\cline { 2 - 6 } & Near-Surge & 1.63 & 1.65 & 0.61 & 0.66 \\
\hline
\end{tabular}

The pressure-ratio and the compressor efficiency are calculated as follows:

Pressure-ratio $(\mathrm{Pr}) \quad=.\quad \mathrm{p}_{02} / \mathrm{p}_{01}$

Efficiency $=\left(\left(\mathrm{p}_{02} / \mathrm{p}_{01}\right)^{(\mathrm{v}-1) / \mathrm{v}}-1\right) /\left(\left(\mathrm{T}_{02} / \mathrm{T}_{01}\right)-1\right)$

where $p_{0}$ is the total pressure, $T_{0}$ is the total temperature, and $\mathrm{Y}$ is the specific heat ratio of 1.4 . The indices 1 and 2 refer the quantities at the inlet and the outlet, respectively.

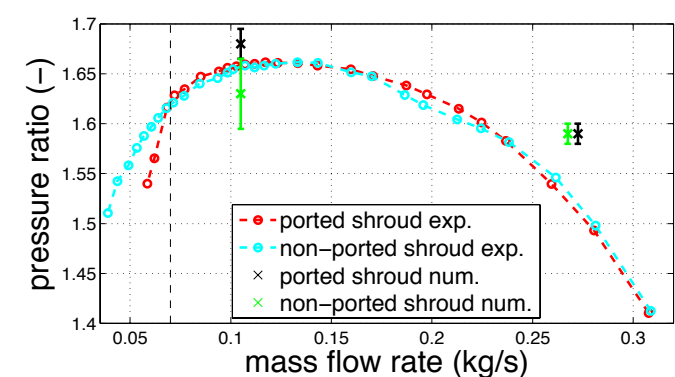

Figure 3. Computational results mapped on the experimentally obtained compressor map for a rotating speed of $64000 \mathrm{rpm}$.

For near optimal efficiency condition, there are no significant differences in the performance parameters between the cases of ported and non-ported shroud. However, the pressure-ratio is higher near-surge than near the optimal operating condition on the constant speed line of $64000 \mathrm{rpm}$. At near-surge the difference between the two configurations becomes apparent. The pressure-ratio is predicted to be higher for the ported shroud compared to the non-ported shroud, whereas the experiments do not show a significant difference in the pressure ratio with and without ported shroud. However, the experiments and the simulations show consistently that the compressor efficiency is less with the ported shroud than without it, as shown in Fig. 4.

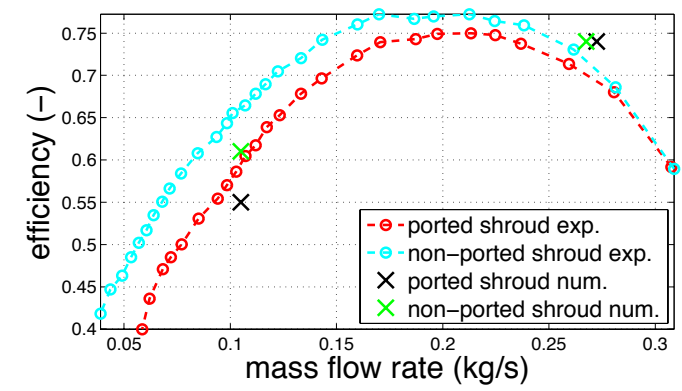

Figure 4. The numerical compressor efficiency estimations are compared to the experimental measurements for a rotating speed of $64000 \mathrm{rpm}$.

The extrema of the monitored pressure-ratio, obtained from the numerical simulation, are indicated in Fig. 3 by error-bars. The variance of the pressure-ratio signal for the ported shroud and the non-ported shroud case is almost equal near the optimal operating condition. Near surge, close to onset of the surge phenomenon, the variance of the pressure-ratio signal is doubled for the non-ported shroud case compared to the ported shroud case. A similar trend in terms of the standard deviation of the outlet pressure has been observed in the experiments [18], as shown in Fig. 5. 


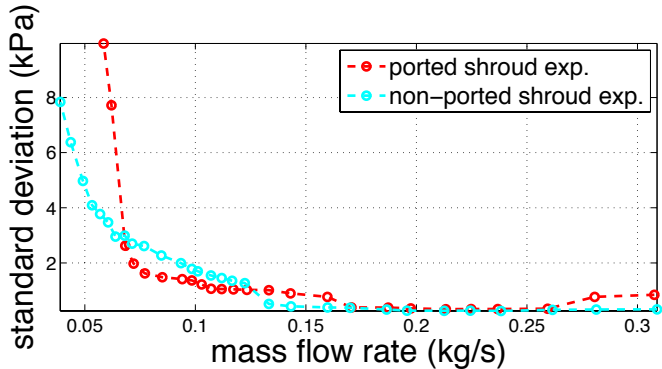

Figure 5. The standard deviation of the experimentally measured outlet pressure signal is shown for a rotating speed of $64000 \mathrm{rpm}$

The numerical simulation data is compared to Particle Image Velocimetry (PIV) measurements [19] performed at planes perpendicular to the compressor inlet axis. Two locations in the inlet region of the compressor have been chosen for the comparison, $3 \mathrm{~mm}$ above and $10 \mathrm{~mm}$ below the ported shroud edge. The PIV measurements have been carried out at slightly different operating conditions for the same speed line of 64000 $\mathrm{rpm}$. The near optimal efficiency condition is compared to a corrected mass flow rate of $0.28 \mathrm{~kg} / \mathrm{s}$ and the near-surge condition is compared to a corrected mass flow rate of 0.1 $\mathrm{kg} / \mathrm{s}$. A bell-mouth inlet has been used at the inlet for the experimental setup, which has not been incorporated in the numerical investigations. About 200 image pairs of the instantaneous PIV pictures have been acquired for each condition to estimate the mean flow field. The laser sheet thickness used for the PIV measurements was about $2 \mathrm{~mm}$.

The comparison of the in-plane velocity field $(\mathrm{m} / \mathrm{s})$ is presented in Fig. 6, where the numerical data is in the left column and the experimental data in the right column. The in-plane velocity field is characterized by the root mean square value of the two velocity components in that plane. For the non-ported shroud cases a consistent agreement can be observed for both conditions, as shown in Fig. $6 a$ in the plane slightly above the ported shroud edge and in Fig. $6 \mathrm{~b}$ in the plane $10 \mathrm{~mm}$ below the ported shroud edge. The ribs geometry has not been cut from the images. Furthermore, the location is close to the impeller and reflections from the solid surfaces in the PIV images at the center where removed. Without the ported shroud, the flow field develops a simple shape, which is captured by the computations and the experimental flow measurements. However, the velocity magnitudes differ slightly, which could be due to the slightly different operating conditions between the experiment and the computations. With the ported shroud the flow field exhibits more flow structures, due to flow recirculation through the ported shroud. Figure $6 \mathrm{c}$ shows the flow field $10 \mathrm{~mm}$ below the ported shroud edge for near optimal efficiency condition with ported shroud. The flow structures that were obtained numerically and experimentally are similar. Both methods predict the flow through the shroud cavity at the same locations. Also the vortical structures in the inlet are predicted at the same locations. However, the velocity magnitudes obtained experimentally are higher, which could be related to the sampling number of snapshots. Moving out of the compressor to the plane $3 \mathrm{~mm}$ above the ported shroud edge, similar velocity magnitudes are observed between experiments and computations. However, discrepancies can be seen at the shroud edge location. In the experimental results, the geometrical contours of the shroud edge can be seen by the circular low velocity region. Also for near surge condition, shown in Fig. 6e, the contours of the shroud edge can be seen.
Thus, the influence of the closely located shroud geometry compared to the investigation plane can be seen in the PIV images. However, the general trends observed for the PIV measurements are captured by the computations. In general it was observed that the simulation results match better with the PIV flow field images taken at slightly lower corrected mass flow rates. Thus, there might be a slight shift between the experimental corrected mass flow rate values and the numerically set mass flow rate values.
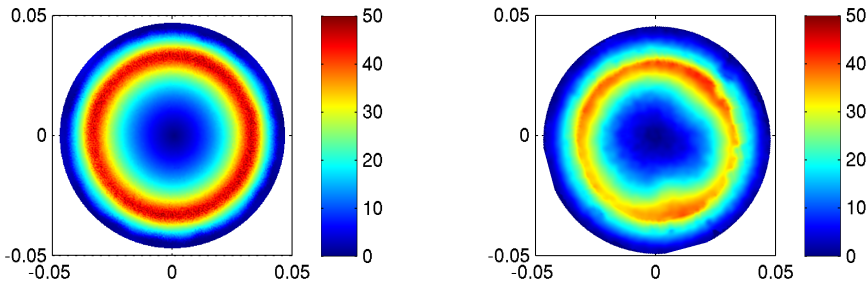

(a) Non-ported Shroud at Near Optimal Efficiency


(b) Non-ported Shroud at Near-Surge


(c) Ported Shroud at Near Optimal Efficiency
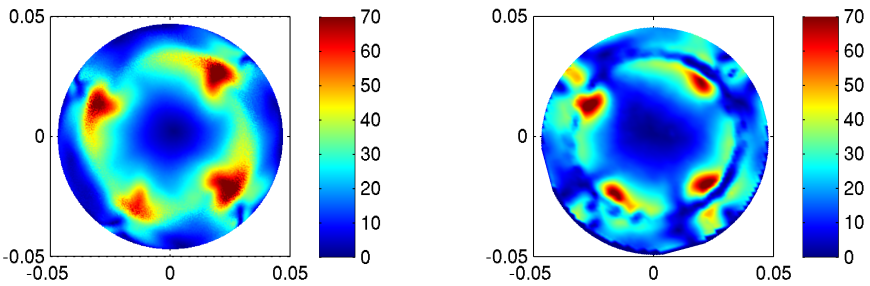

(d) Ported Shroud at Near Optimal Efficiency


(e) Ported Shroud at Near-Surge

Figure 6. The numerical data (left column) is compared to experimental PIV data (right column) in terms of the in-plane velocity magnitude $(\mathrm{m} / \mathrm{s})$ in to planes across the compressor inlet region. The plane shown in a, $\mathrm{d}$, and $\mathrm{e}$, is located $3 \mathrm{~mm}$ above the ported shroud edge, while the plane shown in $b$ and $c$ is located $10 \mathrm{~mm}$ below the ported shroud edge. 
Overall, the comparison between the experimental and the numerical results agree for the ported shroud case. However, near optimal efficiency the pressure-ratio and compressor efficiency are slightly overestimated. This could be due to the smooth and adiabatic wall boundary condition assumption, or due to the fact that the space between the wheel and the back plate has been neglected in the numerical analysis. Sun et. al.[20] carried out numerical work, where he showed that the numerical analysis without modeling the gap, results in an over prediction of the efficiency value. Near surge, the compressor efficiency is underestimated and the pressure ratio is predicted to be different with and without ported shroud. However, the flow structures are constantly captured by the computations and the PIV measurements.

\section{(ii) General Compressor Flow field}

In this section a comparative study of the numerical results has been made between the flow field results obtained from ported and non-ported shroud configuration at near optimal operating condition and near-surge. The flow field of the two configurations at different conditions is required to understand the mechanism that causes the widening of the stable operating range of the compressor.

Starting by examining the near optimal operating condition, Fig. 7 illustrates the time averaged velocity magnitude contours in a mid plane for the two cases, i.e. with ported shroud (Fig. 7a) and with closed ported shroud (Fig. 7b). The location of the plane is shown in Fig. 7c. A nearly symmetric, homogeneous flow pattern is observed for both cases, although the ported shroud cavity is not symmetric. For the case with closed ported shroud, the port slots are closed at the interface to the wheel, while the geometry of the shroud is still present. Thus, very low velocities are observed in the shroud cavity for the non-ported shroud case. At the blade tips of the wheel, the flow is accelerated to high velocities, which causes the flow to separate downstream in the blade passage. However, for the case with ported shroud at near optimal efficiency condition, a substantial amount of flow exits through the shroud cavity (Fig $7 a)$. The flow recirculating from the shroud cavity to the compressor inlet disturbs the inflow of the wheel passage by deviating the flow towards the compressor axis. This can be seen by the decreased amount of flow separation at the hub entry. However, for the near optimal efficiency condition, the flow is more uniform at the wheel inlet as there is no blockage to the flow.

The flow field close to the slot leading to the shroud cavity is shown in Fig. 8 for a time instant near optimal efficiency. Although the flow field exhibits only some inhomogeneous flow motion, a significant amount of flow is recirculated to the compressor inlet through the ported shroud. It can be observed that the flow is deviated by the flow separation occurring at the compressor inlet. The flow is deflected from the inlet walls at different angles for the cases with and without ported shroud.
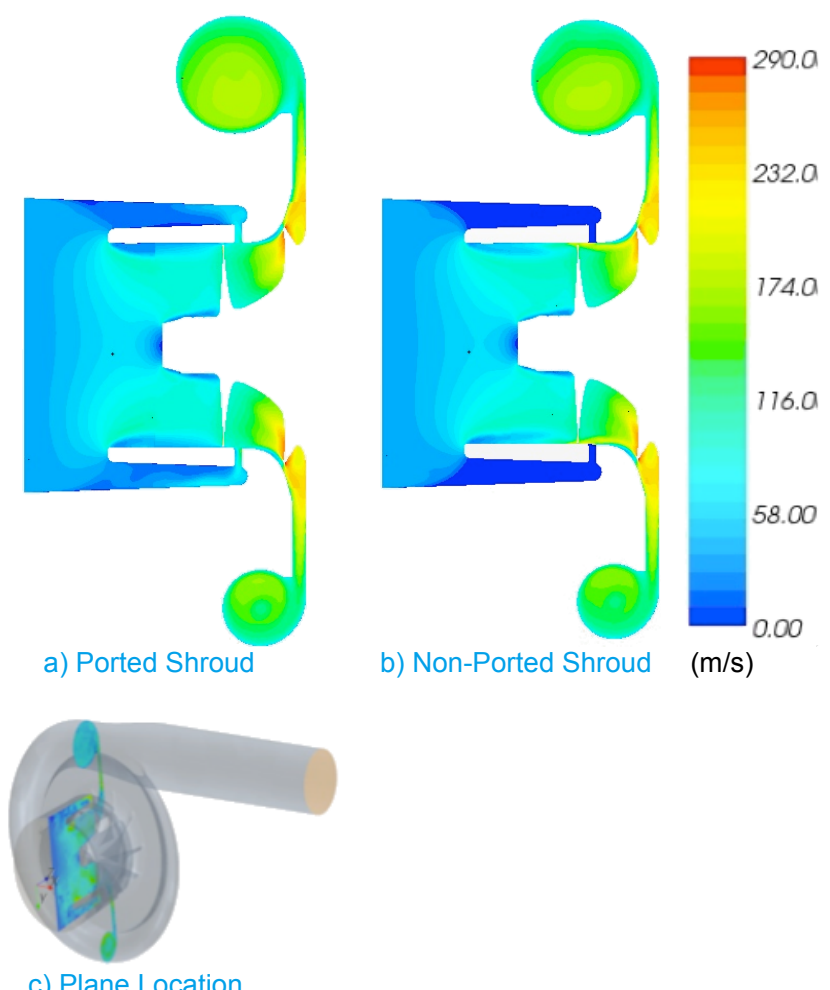

b) Non-Ported Shroud

$(\mathrm{m} / \mathrm{s})$

c) Plane Location

Figure 7. The time averaged velocity magnitude $(\mathrm{m} / \mathrm{s})$ contours are shown at the optimal efficiency operating condition for the ported and non-ported shroud configurations.

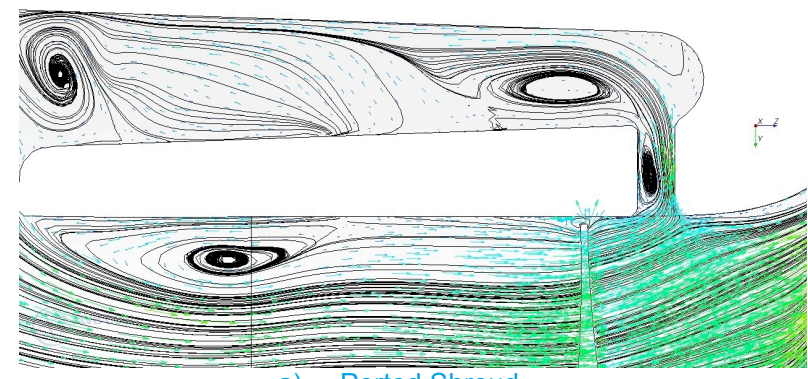

a) Ported Shroud

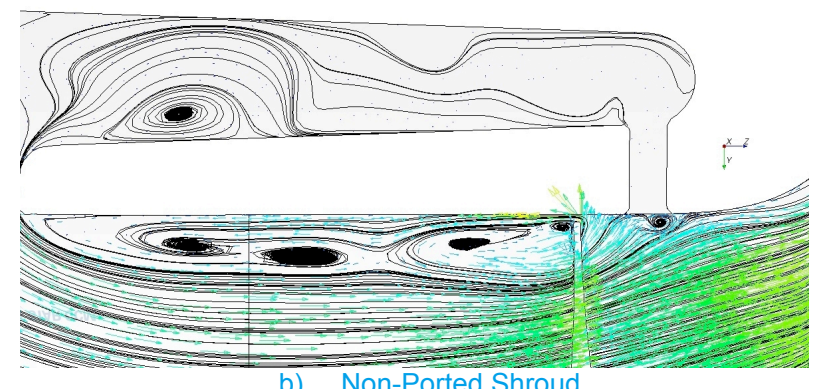

Figure 8. The streamlines with the underlaid velocity vectors illustrate the instantaneous flow field in the surrounding region of the slot to the shroud cavity near optimal efficiency operating condition.

In a mid plane cut through the diffuser, the time averaged velocity magnitudes near optimal efficiency and the near surge with ported shroud are shown in Fig. 9. The velocities in the wheel passages are of similar magnitudes for the two conditions, since the wheel rotates with the same rotational 
speed. However, the velocity magnitudes in the wheel are higher for near optimal efficiency. In the diffuser, the volute, and the exit pipe, the velocity magnitudes are higher at the near optimal efficiency compared to near surge, since the exit static pressure is higher for near surge. A difference in the velocity contours shown in Fig. 9 between the operating conditions can be noted. The velocity contours of near optimal efficiency exhibit a slight increase of the velocity in the diffuser in clockwise direction from the tongue following the volute. However, for near surge, a velocity magnitude increase at the 2 O'clock to 3 O'clock location is visible, while the velocity magnitudes thereafter in clockwise direction are lower. A the 6 O'clock location near surge, the velocity magnitudes at the outer volute wall are higher than in the center of the volute, which indicates a high amount of helical flow motion in the volute.

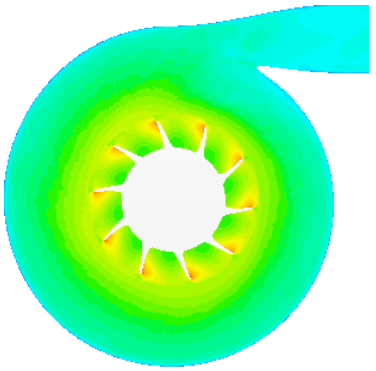

a) Near Optimal Efficiency



b) Near Surge

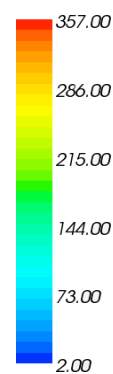

$(\mathrm{m} / \mathrm{s})$
Figure 9. The time averaged velocity magnitude $(\mathrm{m} / \mathrm{s})$ contours are shown at near optimal efficiency and the near surge for the ported shroud configuration.

The time averaged static pressure contours near optimal efficiency and near surge condition with ported shroud exhibit the consequence of the velocity distribution. As illustrated in Fig. 10b, a sharp pressure gradient develops under the tongue near surge operation condition. Where the high velocities have been visible, a low pressure region is shown in Fig. 10b. Contrary to near surge condition, the static pressure contours are skewed towards the 11 O'clock location for near optimal efficiency.

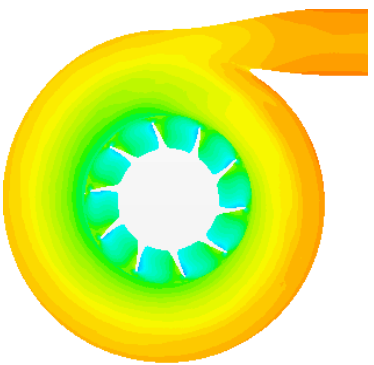

a) Near Optimal Efficiency



b) Near Surge

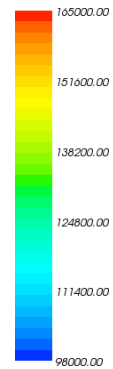

$(\mathrm{Pa})$
Figure 10. The time averaged static pressure $(\mathrm{Pa})$ contours are shown at near optimal efficiency and the near surge for the ported shroud configuration.

Figure 11 shows the time averaged axial velocity contours for near optimal efficiency and near-surge at a location close to the exit of the shroud cavity. This location is chosen, since significant changes in the turbulence quantities are expected between the cases during off-design condition. The previous comparison of the flow-field at near optimal efficiency condition for the ported and non-ported shroud case revealed a nearly symmetric flow pattern at the inlet of the compressor and in particular near to the shroud cavity exit. However, the flow field through the shroud cavity is not symmetric as the ribs in the shroud cavity are arranged in an asymmetric manner. Figure 9a shows that in this plane the axial flow velocities with ported shroud are higher for both operating conditions. High velocity jet-like flow structures are generated by the flow interacting with the ribs. Near-surge, more flow streams through the shroud cavity, as visible in Fig. 11, where the negative axial velocity is increased, compared to near optimal efficiency condition. The work delivered by the wheel may not be enough to transport the flow at the required exit pressure to the outlet. However, for the non-ported shroud the velocity contours show an expected axial symmetric flow pattern.

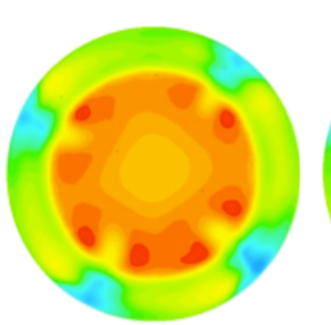

Ported Shroud

(a) Near Optimal Efficiency



Non-Ported Shroud

\section{(c) Near Optimal Efficiency}

(d) Near-Surge

(b) Near-Surge

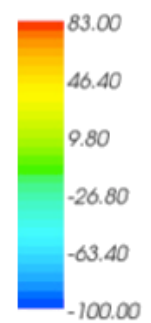

$(\mathrm{m} / \mathrm{s})$
Figure 11. The time averaged axial velocity $(\mathrm{m} / \mathrm{s})$ contours are shown for ported and non-ported shroud at near optimal efficiency and nearsurge. The inflow towards the compressor is indicated by positive values, whereas the negative values represent backflow.

The velocity flow field for a cell volume can be decomposed into a time-averaged mean velocity and a time fluctuating part (the absolute value of the time-averaged mean velocity minus the instantaneous velocity), which corresponds to the variance of the velocity. The axial velocity fluctuations are shown in Fig. 12. At near optimal operation the velocity fluctuations are higher for the ported shroud than for the non-ported shroud. This is expected since the flow originating from the shroud cavity mixes with the incoming main flow at the cavity exit. Near surge, the turbulence levels increase for both configurations to a similar magnitude. The fluctuations are more intense at the ribs location, where jet-like structures form. 


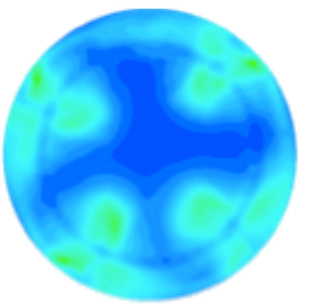

Ported Shroud


$(\mathrm{m} / \mathrm{s})$

(a) Near Optimal Efficiency

(b) Near-Surge
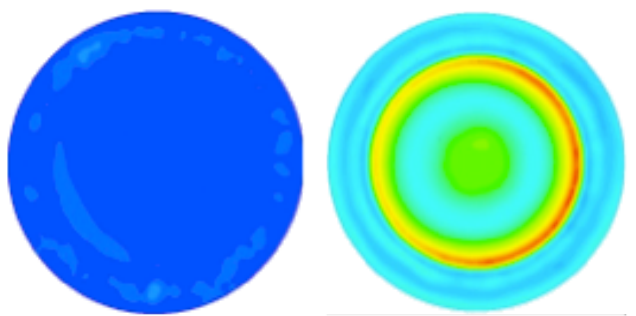

Non-Ported Shroud

(c) Near Optimal Efficiency

(d) Near-Surge

Figure 12. The velocity fluctuations $(\mathrm{m} / \mathrm{s})$ for ported and non-ported shroud case are shown at near optimal efficiency and near-surge.

For the non-ported shroud, the flow is highly non-uniform at the wheel inlet. In particular on the shroud side, high velocity fluctuations throughout the periphery are observed. The high velocity fluctuations are caused by the flow recirculation, which arises due to reduction in the inlet mass-flow for the constant rotational speed. The recirculation region is trapped in the blade passage and acts as a blockage to incoming main flow. This affects the blade loading near the inducer section and the incoming incidence of the flow at the leading edge. Thereby, the work performed by the impeller is reduced leading to the generation of a lower pressure-ratio. Thus, a lower pressureratio is induced by the inefficient blade loading caused by the unsteady flow motion for the non-ported shroud near-surge.

The incidence angle alteration of the flow entering the blade passage at different operating conditions with ported shroud is shown in Fig. 13. At near optimal efficiency, the blade passage is entirely utilized and a flow separation from the blades is not visible. Contrary to near-surge, the flow enters at a much shallower angle into the blade passage and flow separation occurs on the blades. Moreover, the flow upstream of the blade passage is highly disturbed and the inhomogeneous flow structures propagate into the blade passages.

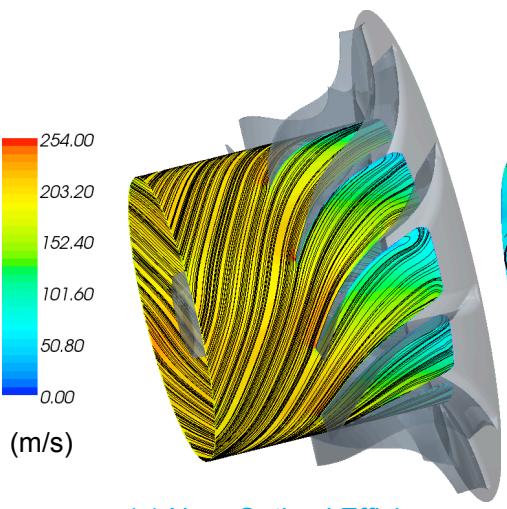

(a) Near Optimal Efficiency

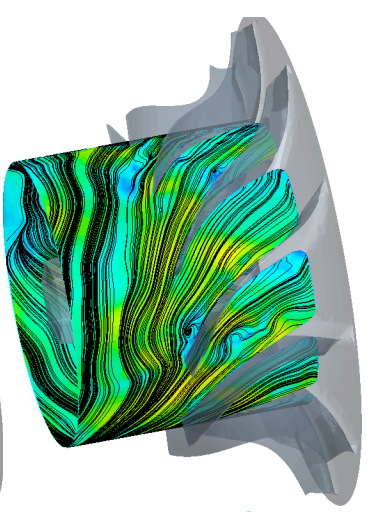

(b) Near-Surge
Figure 13. Instantaneous streamlines are shown on a cylindrical surface colored by the velocity magnitude $(\mathrm{m} / \mathrm{s})$ for near optimal efficiency and near surge with ported shroud.

The quantitative plot for the time averaged axial and tangential velocities is shown in Fig. 14 on the symmetry line of the plane shown in Fig 11e. A comparison of the design operation for the two configurations reveals that the flow through the non-ported shroud case is symmetric, as there is hardly any flow motion inside the shroud cavity. However, for the ported shroud a stream develops through the shroud cavity even at design condition and the flow is not symmetric at the shroud cavity exit.

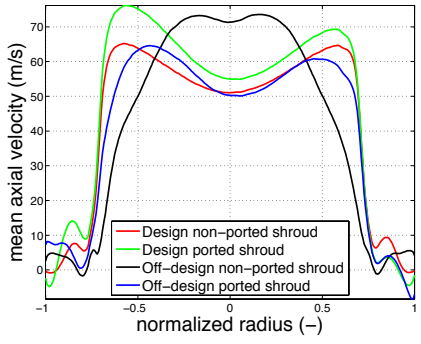

a) Mean Axial Velocity



b) Mean Tangential Velocity
Figure 14. Axial and tangential velocity $(\mathrm{m} / \mathrm{s})$ plots are shown at the vertical symmetry line location indicated in Fig. 11e for the ported and non-ported shroud at design condition.

The advantage of the ported shroud cavity over the non-ported shroud configuration becomes clear for the off design condition, e.g. near-surge. With the ported shroud, the inlet blockage due to flow separation is removed through the shroud cavity. The flow disturbances are not removed for the nonported shroud case and this results in blocking of the flow near the wheel inlet. The flow recirculation in the inlet region reduces the effective flow area and this in turn increases the flow velocity. This affects the incidence of the flow into the blade passage, which causes flow separation and the formation of a backflow region. In the tangential velocity plot, the same trend is observed as that of the mean axial velocity. For the design condition case, the tangential velocity is low for both configurations. Near-surge the tangential velocity increases in both cases, but the velocity is comparatively high for the non-ported shroud.

Streamline visualizations for the two cases at near-surge are shown in Fig. 15. The flow is highly disturbed in the inlet region for both cases. For the non-ported shroud case, large 
recirculating flow structures and flow separations are visible, which block the entrance to the wheel and propagate far into the wheel passage. The flow near the inducer is highly nonuniform and this results in an improper blade loading of the leading edge section. This situation affects the flow entering the impeller. The disturbances are even visible in the diffuser and the volute of the compressor. Even the velocity fluctuations at the shroud exit location are high for the nonported shroud (see also Fig. 12d). With ported shroud, enhanced flow through the shroud cavity is visible, which removes flow disturbances from the wheel passages. Therefore, the streamline visualization exhibits a more uniform flow through the wheel passage for the ported shroud. The blockage due to flow disturbances is not visible for this case. Thus, the static pressure difference due to high loading causes the flow to move towards the shroud cavity and clears the way to the incoming main flow and thereby increases the stability of the compressor.

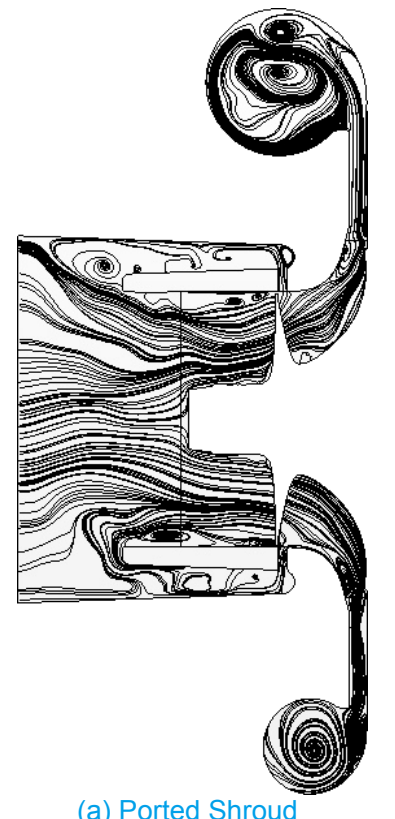

(a) Ported Shroud

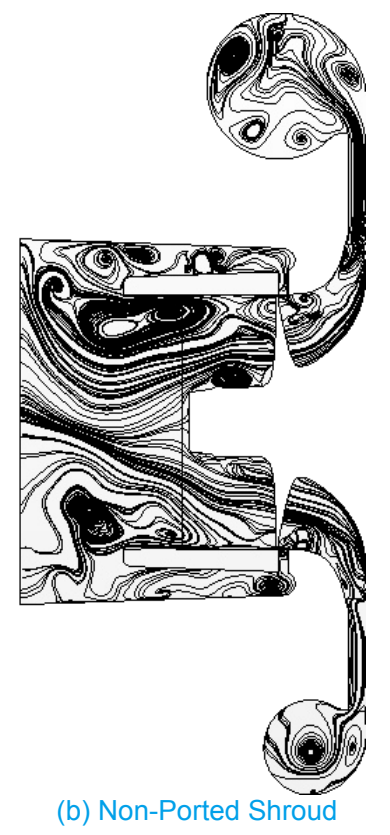

Figure 15. Streamline visualizations illustrate the instantaneous unsteady flow for the ported and non-ported shroud near-surge.

\section{Shroud Cavity Flow}

Generally, the ported shroud improves the flow through the wheel passage by clearing the stagnant flow and the blocking flow structures near the wheel inlet region through the ported slot. The removed flow passes through the shroud cavity towards the compressor inlet. Although the ported shroud stabilizes the flow, it is effective only if the losses occurring inside the ported shroud are minimized. The backflow that occurs during the off-design condition due to the high pressure-ratio and thus, the increased pressure gradient affects the blade loading near the leading edge. This may result in hindrance for the impeller to produce the required pressure rise. The pressure balance [21] across the ported shroud is given by the following equations:

$\mathrm{p}_{\text {0port }}-\Delta \mathrm{p}_{\text {(shroud cavity - loss) }}>\mathrm{p}_{\text {0wheel }}$ $\mathrm{p}_{\text {Oport }}-\mathrm{p}_{\text {Owheel }}=\Delta \mathrm{p}_{\text {(Leading edge }- \text { gain })}$

$\Delta \mathrm{p}_{\text {(Leading edge - gain) }}>\Delta \mathrm{p}_{\text {(shroud cavity - loss) }}$

Where $p_{0 \text { wheel }}$ is the total pressure at the wheel inlet, $p_{0 p o r t}$ is the total pressure at the port inlet, $\Delta \mathrm{p}_{\text {(shroud cavity - loss) }}$ is the pressure loss through the shroud cavity, and $\Delta p_{\text {(Leading edge - gain) }}$ is the pressure gain through the inducer section. The corresponding values obtained by the numerical simulations are the following: $\mathrm{p}_{\text {0port }}=133375 \mathrm{~Pa}, \mathrm{p}_{\text {owheel }}=100620 \mathrm{~Pa}, \Delta \mathrm{p}_{\text {(shroud cavity }- \text { loss) }}=$ $29858 \mathrm{~Pa}$, and $\Delta \mathrm{p}_{\text {(Leading edge - gain) }}=32756 \mathrm{~Pa}$.

The required condition for an effective performance of the ported shroud is that the total pressure loss across the shroud cavity should be less than the total pressure rise that is produced by blade loading between the wheel inlet and the port inlet. In the present simulation, the required condition is met with this ported shroud cavity indicating that the total pressure rise between wheel and port inlet is higher. The reduction in the $\Delta \mathrm{p}_{\text {(shroud cavity - loss) }}$ can be achieved by having a guided flow inside the cavity, e.g. using vanes.

The LES results of the performance parameter show that the compressor efficiency of the non-ported shroud case is higher than the ported shroud. Although the required pressure balance is met with the present ported shroud casing, this is also associated with thermal and frictional losses induced by the flow through the shroud cavity. As explained in the previous section, the flow accelerates across the shroud cavity and mixes with the flow from the inlet. At the cavity exit, the axial velocity fluctuations are increased and the cavity ribs results in generating jet-like structures, as shown in Fig. 16 and these structures induce an intense mixing of the flow. The jetlike structures exhibit a high velocity and therefore the structures penetrate quite far out of the shroud cavity into the inlet region of the compressor. The structures jutting out from the shroud cavity are bent by the compressor inlet flow back into the inducer and move towards the wheel. This can be seen in Fig. 17, where the in-plane velocity vectors are shown on six consecutive cross-planes. This flow pattern results in a turbulent mixing loss. However, the flow through the shroud cavity can be guided with vanes to decelerate the flow and thereby reduce the mixing loss induced by the ported shroud.


Figure 16. The axial velocity $(\mathrm{m} / \mathrm{s})$ component is shown to illustrate the jet-like flow structures at two time instants in the plane specified in Fig. $11 \mathrm{e}$. The periphery of the shroud and the compressor are indicated by black circles. Positive values represent inflow into the compressor, whereas the negative values are backflow. 


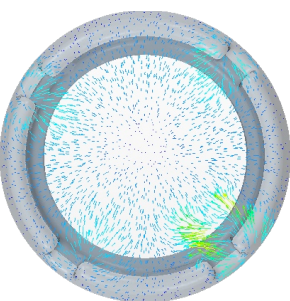

(a)

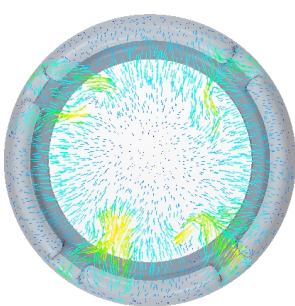

(d)



(b)

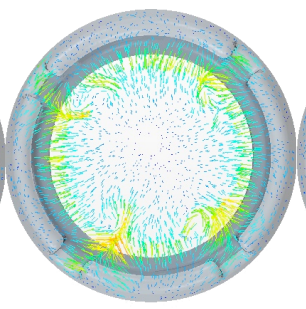

(e)

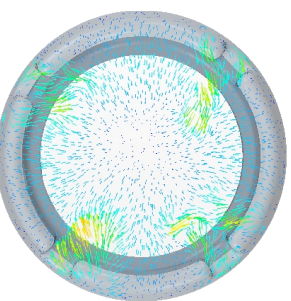

(c)

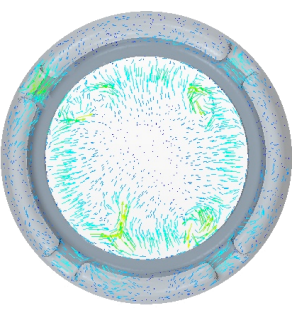

(f)
Figure 17. The in-plane velocity vectors are shown for six planes moving consecutively into the compressor from a to f. The planes range equidistantly in between the red planes indicated in Fig. 11e. The view is towards the compressor.

\section{Frequency Spectra}

The governing frequencies induced by the unsteady flow phenomena can be determined by evaluating the recorded signal in monitoring probes. The spectra of the signal can be used to identify the inherent unsteady flow phenomena. The flow unsteadiness at near surge is expected to be prominent in the diffuser region. The power spectra density is shown in Fig. 18 for the recorded tangential velocity component signal in the diffuser. The blade passage frequency is visible in the spectra in the form of a sharp peak at $10.6 \mathrm{kHz}$ along with one harmonic. Additionally, a second hump in the range of about $530 \mathrm{~Hz}$ (about half of the frequency of the rotor $1060 \mathrm{~Hz}$ ) is also predicted for both configurations and could arise from the occurrence of the rotating stall instability.

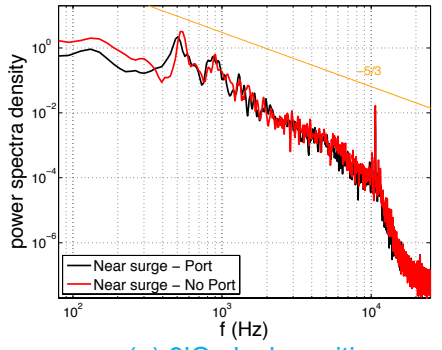

(a) 9'O clock position

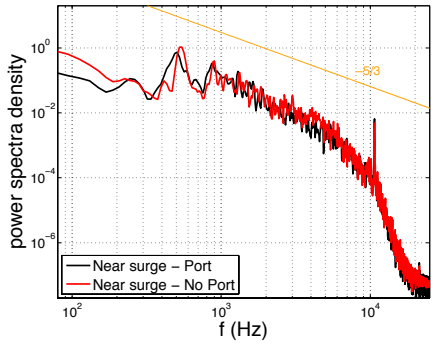

(b) 3'O clock position
Figure 18. The power spectra density of the tangential velocity component are shown at the locations (a) 9'O clock position (b) 3'O clock position (under the tongue) in the diffuser.

Additionally, the frequency spectra indicate that a sufficient numerical mesh resolution has been used. Figure 18 shows the Kolmogorov's characteristic $-5 / 3$ slope of the turbulent kinetic energy dissipation. About an order of magnitude of the inertial subrange is captured by the computational mesh grid and no spurious energy buildup in the high frequency range occurs.

Page 10 of 11
Four ribs are used to support the ported shroud casing. It was reported in literature [21] that the presence of ribs could result in a high cycle fatigue of the impeller inducer blade. This high cycle fatigue is a non-linear fluid-structure interaction problem, where the origin of the cause is not completely known. With respect to a ported shroud casing, the frequency of the excitation force is given by the order of harmonics of the rib excitation times the number of ribs times the rotating frequency of the rotor. Thus, the ribs are arranged in an asymmetric manner, which reduces the amplitude of low order harmonics. This is one possible solutions to reduce the high cycle fatigue. In the present case, this excitation frequency occurs around $4240 \mathrm{~Hz}$ for the first order harmonics.

\section{Conclusions}

The flow phenomena occurring with a ported and a non-ported shroud in an automotive turbocharger centrifugal compressor during near optimal efficiency operating condition and nearsurge were analyzed using LES with sliding mesh. The performance parameters, i.e. a compressor efficiency and pressure-ratio, of the centrifugal compressor were calculated and compared with experimental data. Agreement between the numerical and experimental results was obtained and the trends could be predicted consistently with the experiments.

The flow fields for the two operating conditions were analyzed for the ported and a non-ported shroud. Near optimal efficiency, the ported and non-ported shroud configurations achieved similar performance parameter values. However, a significant amount of flow recirculating through the ported shroud was observed even for near optimal efficiency. Thus, the flow-field exhibits a different behavior even for stable operating condition.

For off-design condition, the ported shroud decreased the compressor efficiency. However, the amount of flow fluctuations was significantly reduced with the ported shroud, since the unsteady flow was recirculated through the ported shroud. Thus, extension of the compressor lifetime due to the ported shroud is expected. With flow recirculation, high velocity jet-like structures were generated by the ribs increasing the mixing at the shroud cavity exit. It has been shown that the lower efficiency with ported shroud at near surge occurs due the mixing losses at the shroud cavity exit. The high blade loading near the leading edge and a better flow through the blade passage, leads to a higher pressure ratio for the ported shroud compared to the non-ported shroud. An increased amount of back flow and unsteady flow motion was observed for the non-ported shroud at the wheel inlet affecting the blade loading on the leading edge section of blade. This modifies the flow incidence at the entry, which in turn reduces the pressure ratio. The flow disturbances propagate through the entire compressor domain and cause increased variation of the performance parameters for the non-ported configuration.

The present results identify the jet-like structures generated by the recirculating flow through the shroud cavity as limitation of the compressor efficiency for the ported shroud configuration at the simulated off-design operation condition. This suggests that improved the efficiency of the ported shroud can be achieved by using guiding vanes in the shroud cavity as has been proposed in previous literature. These changes can lead to a decrease in the flow velocities of the jet-like structures and 
thereby reducing the mixing losses induced by these structures. The jet-like structures should be decreased in strength to decrease the penetration length into the inlet of the compressor.

\section{References}

1. Rodgers, C., "Centrifugal Compressor Inlet Guide Vanes for Increased Surge Margin," J. Turbomach. 113(4):696702, 1991, doi:10.1115/1.2929136

2. Whitfield, A., and Abdullah, A.H., "The Performance of a Centrifugal Compressor with High Inlet Prewhirl," J. Turbomach. 120(3):487-493, 1998, doi:10.1115/1.2841744

3. Ishida, M., Sakaguchi, D., and Ueki, H., 2006, "Effect of Pre-swirl on Unstable Flow Suppression in a Centrifugal Impeller With Ring Groove Arrangement," ASME Turbo Expo GT2006-90400, 2006, doi:10.1115/GT2006-90400

4. Barton, M. T., Mansour, M. L., Liu, J. S., and Palmer, D. L., "Numerical Optimization of a Vaned Shroud Design for Increased Operability Margin in Modern Centrifugal Compressors," J. Turbomach. 128:627-631, 2004, doi: 10.1115/1.2187526

5. Saha, S. L., Kurokawa, J., Matsui, J., and Imamura, H., "Passive Control of Rotating Stall in a Parallel-Wall Vaned Diffuser by J-Grooves," J. Fluids Eng. 123(3):507-515, 2001.

6. Macdougal, I., and Elder, R. L., "The Improvement of Operating Range in a Small, High Speed, Centrifugal Compressor Using Casing Treatment," IMechE Conference on Turbochargers and Turbocharing C32/82, 1982.

7. Yamaguchi, S., Yamaguchi, H., Goto, S., Nakao, H., and Nakamura, F., "The Development of Effective Casing Treatment for Turbocharger Compressor," Proceedings of the $7^{\text {th }}$ IMechE International Conference on Turbocharger and Turbocharging, London C602/016/2002, May 14-15, 2002.

8. Zheng, X., Zhang, Y., Yang, M., Bamba, T., and Tamaki, $\mathrm{H}$., "Stability Improvement of High-Pressure-Ratio Turbocharger Centrifugal Compressor by Asymmetrical Flow Control - Part II: Nonaxisymmetrical SelfRecirculation Casing Treatment," J. Turbomach. 135(2): 021007-1 - 021007-8, 2002, doi:10.1115/1.4006637

9. Subenuka Sivagnanasundaram, Stephen Spence, Juliana Early and Bahram Nikpour, "An impact of Various Shroud Bleed Slot configurations and Cavity Vanes on Compressor Map Width and the Inducer Flow Field", J. of Turbomach. 135(4), 2013, doi:10.1115/1.4007513.

10. Ishida, M., Sakaguchi, D., and Ueki, H., "Effect of pre-swirl on unstable flow suppression in a centrifugal impeller with ring groove arrangement", ASME Turbo Expo GT200690400, 2006, doi:10.1115/GT2006-90400

11. Hellström, F., Gutmark, G., and Fuchs, L., "Large Eddy Simulation of the Unsteady flow in a Radial Compressor Operating Near Surge," J. of Turbomach. 134(5):11571169, 2012, doi:10.1115/1.4003816.

12. Guleren, K.M., Afgan, I., and Turan, A., "Predictions of Turbulent Flow for the Impeller of a NASA Low-Speed Centrifugal Compressor," J. Turbomach. 132(2):021005-1 - 021005-8, 2010, doi:10.1115/1.3140824.

13. Fisher, F. B., "Application of Map Width Enhancement Devices to Turbocharger Compressor Stages," SAE Trans., 97(6):1303-1310, 1989.

14. Hunziker, R., Dickmann, H. P., and Emmrich, R., "Numerical and Experimental Investigation of a Centrifugal Compressor with an Inducer Casing Bleed System," J. Power Energy, 215(6): 783-791, 2001.

15. Guleren, K.M., Turan, A., and Pinarbasi A., "Large Eddy Simulation of the flow in a Low-Speed Centrifgual Compressor," Int. J. Numerical Methods in Fluids, 56:1271-1280, 2008, doi: 10.1002/fld.1741.

16. Chow, P., Cross, M., and Pericleous, K., "A natural extension of the conventional finite volume method into polygonal unstructured meshes for CFD application," Appl. Math. Modelling 20(2):170-183, 1996, doi: 10.1016/0307904X(95)00156-E.

17. STARCCM+, Commercial code, Ver. 6.06, CD-adapco, Melville, NY (1987).

18. Guillou, E., Gancedo, M., Gutmark, E., and Mohamed, A., "PIV investigation of the flow induced by a passive surge control method in a radial compressor," Experiments in Fluids, 53(3):619-635, 2012.

19. Guillou, E., DiMicco, R., Gutmark, E., Mohamed, A. et al., "Characterization of a Ported Shroud Compressor using PIV Measurements," SAE Technical Paper 2010-01-1225, 2010

20. Sun, Z., Tan, C., and Zhang, D., "Flow field structures of impeller backside cavity and its influence on the centrifugal compressor," Proceedings of ASME TurboExpo, June 8-12, USA, 2009

21. Chen, H., and Lei, V., "Casing Treatment and Inlet Swirl of Centrifugal Compressors," J. of Turbomach, 135(4): 041010-1 - 041010-8, 2013, doi:10.1115/1.4007739

\section{Acknowledgments}

The Competence Center for Gas Exchange (CCGEx) and the Swedish Energy Agency (STEM) are acknowledged for supporting this project. This work was supported by the Swedish National Infrastructure for Computing (SNIC 002-1211 \& SNIC 2013-11-19) via HPC2N and PDC. 